\title{
Chronic Tattoo Reactions Cause Reduced Quality of Life Equaling Cumbersome Skin Diseases
}

\author{
Katrina Hutton Carlsen • Jørgen Serup \\ Department of Dermatology, 'Tattoo Clinic', Bispebjerg University Hospital, Copenhagen, Denmark
}

\begin{abstract}
Tattoos are often associated with mild complaints, but some people develop complications that may require medical treatment, and the burden of these events has hitherto been neglected. To understand the dimensions and the psychological symptomatology of adverse events both the sensory and affective impacts, including the effect on quality of life, should be studied. Itch severity and influence on quality of life can be measured objectively. The Itch Severity Scale and Dermatology Life Quality Index scoring systems have been applied to different dermatological diseases. When ISS and DLQI scores were applied to patients with chronic tattoo reactions, tattoo complaints and impact on quality of life that were comparable to patients presenting cumbersome dermatological disease such as psoriasis, eczema and pruritus, which often show widespread effects to the skin, were uncovered. In conclusion, chronic tattoo reactions should be ranked as a cumbersome dermatological disease and, accordingly, given priority attention and qualified treatment by the public health care system.
\end{abstract}

() 2015 S. Karger AG, Basel

\section{Introduction}

Signs and symptoms of tattooed skin deviating from normal skin may be mild and acceptable, i.e. at the level of complaint or, when more severe and cumbersome, considered a complication, both of which may require medical treatment. The concept of disease goes beyond the objective signs and findings; it also includes the subjective experience and its influence on patients' lives.

Itch, stinging and pain are the most frequent reported tattoo complaints $[1,2]$. Sun-induced reactions, especially in dark colours, are reported in one of five tattooed individuals, with swelling and itching being the most frequent complaints.

The chronic complications are dominated by allergic reactions, which may develop within weeks, months or years. Allergic reactions especially occur in red tattoos. Patients presenting clinically with allergic reactions are patch tested with common allergens, textile dyes, a battery of suspect tattoo ink stock products and their individual suspect inks, all 
of which result in an overall negative outcome [3]. The putative allergens are not directly present in the ink stock products but are formed inside the dermis over a longer period.

In this chapter, we will review the subjective symptoms associated with chronic tattoo complaints, i.e. reactions when the tattooed person may seek help from a medical professional. How do these symptoms intrude on patients' lives? What effects do they play on quality of life?

\section{Measurements of Itch and Quality of Life}

Different methods are employed to measure the burden of disease and the impact on quality of life. In the field of dermatology, the Itch Severity Scale (ISS) and the Dermatology Life Quality Index (DLQI) are mainly used.

The ISS is a questionnaire designed for the patient [4]. It was devised by Majeski et al. [5] to measure both the sensory and the affective dimensions of itch and is composed of the following 7 topics:

(1) description; (2a) sensory; (2b) affective; (3) body area; (4) intensity; (5) effect on mood; (6) effect on sexual desire/function, and (7) effect on sleep.

DLQI, which was introduced by Finlay and Khan, is a questionnaire including the following topics and elements. (1) and (2): Symptoms and feelings; (3) and (4): daily activities; (5) and (6): leisure; (7): work and school; (8) and (9): personal relationships, and (10): treatment $[6,7]$.

Both scoring systems are self-explanatory and can easily be completed by an adult patient. They have also both been evaluated on dermatological patients and concluded to be valid $[8,9]$. Higher scores in the ISS and DLQI systems indicate a greater impact on the patients' lives. By utilizing the same methods for interpreting patients' experiences with disease, a comparison of different disease populations can be performed and the best therapy can be implemented.

\section{Skin Disease and Quality of Life}

Itch is a great burden for many dermatological patients influencing their psyche and, thereby, their ability to cope with disease. The ISS has been used to evaluate patients with pruritus, genital pruritus and nephrogenic pruritus as well as atopic dermatitis, psoriasis and urticaria $[5,8]$, which revealed ISS scores from 7.4 to 13.4 (table 1 ). Besides intensive itching, the patients also experienced depressive symptoms, anxiety and sleep impairments, which in some cases had a substantial effect on their daily life.

The DLQI has also been utilized on patients with hand eczema, pruritus and neurodermatitis and has demonstrated a moderate effect on patients' lives $[4,10,12]$. Psoriasis and atopic dermatitis have a big effect on patients' lives (table 1) [11-14].

\section{Tattoo Complications and Quality of Life}

Patients with tattoo reactions were often reported to experience itch/stinging at an extreme level when at its worst that commences all hours of the day (morning/afternoon/evening/night) [15].

Patients with tattoo reactions revealed an average ISS score of 7.2, with a range from 0 to 21 [15]. The influence of itch is presented in figure 1.

Itch was associated with concern in $48 \%$ of cases, irritation in $18 \%$ of cases, depression in $20 \%$ of cases, anxiety in $43 \%$ of cases, and concentration problems in $48 \%$ of cases. Itch also affected patients' sleep, ranging from problems falling asleep to sleep disturbance.

Interestingly, the grade of the symptom was independent of the size of the afflicted area.

The average DLQI score was 7.4 [15]. Tattoo problems had a moderate effect on patient's quality of life, and the results of the influence of tattoos on daily living are presented in figure 2 . Symptoms and feelings had the greatest impact 


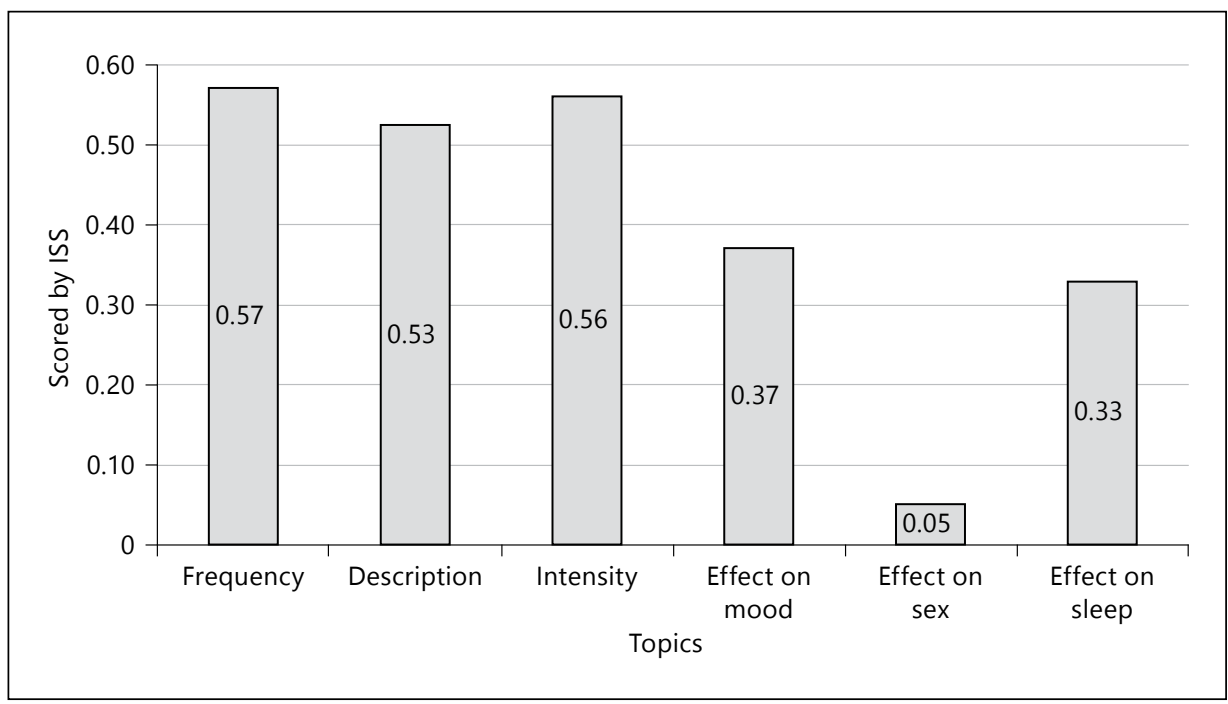

Fig. 1. The extent of tattoos causing itch, measured by the Itch Severity Scale (ISS).

Table 1. Dermatological diagnoses scored by the Itch Severity Scale (ISS) and the Dermatology Life Quality Index (DLQI)

\begin{tabular}{lcccl}
\hline Diagnoses & $\begin{array}{l}\text { DLQI score, } \\
\text { in total }\end{array}$ & $\begin{array}{l}\text { ISS, } \\
\text { in total }\end{array}$ & $\begin{array}{l}\text { Number of } \\
\text { participants } \\
\text { (n) }\end{array}$ & Reference \\
\hline Hand eczema & 8.0 & - & 416 & Agner et al. [10] \\
Pruritus & 8.8 & - & 40 & Zachariae et al. [4] \\
& - & 9.7 & 83 & Zachariae et al. [8] \\
Genital pruritus & - & 7.4 & 93 & Majeski et al. [5] \\
Nephrogenic pruritus & - & 8.6 & 12 & Zachariae et al. [8] \\
Atopic dermatitis & - & 8.5 & 11 & Zachariae et al. [8] \\
& 10.7 & 9.5 & 20 & Zachariae et al. [8] \\
Neurodermatitis & 9.34 & - & 415 & Kim et al. [11] \\
Psoriasis & 13.32 & - & 250 & An et al. [12] \\
& - & 7.5 & 20 & Zn et al. [12] \\
& 13.1 & - & 57 & Kachariae et al. [8] \\
Urticaria & 10.0 & & 250 & Tang et al. [14] [1] \\
\hline
\end{tabular}

on patients' lives, including itching, soreness, pain and/or stinging symptoms. More than half of the patients experienced varying degrees of embarrassment/self-consciousness due to their tattoo reactions. Tattoo reactions also influenced daily activities, e.g. housekeeping in $38 \%$ of cases, choice of clothing in $53 \%$ of cases and leisure, e.g. sport activities, in $40 \%$ of cases. Personal relationships, including sex, were only mildly influenced (13\%). 


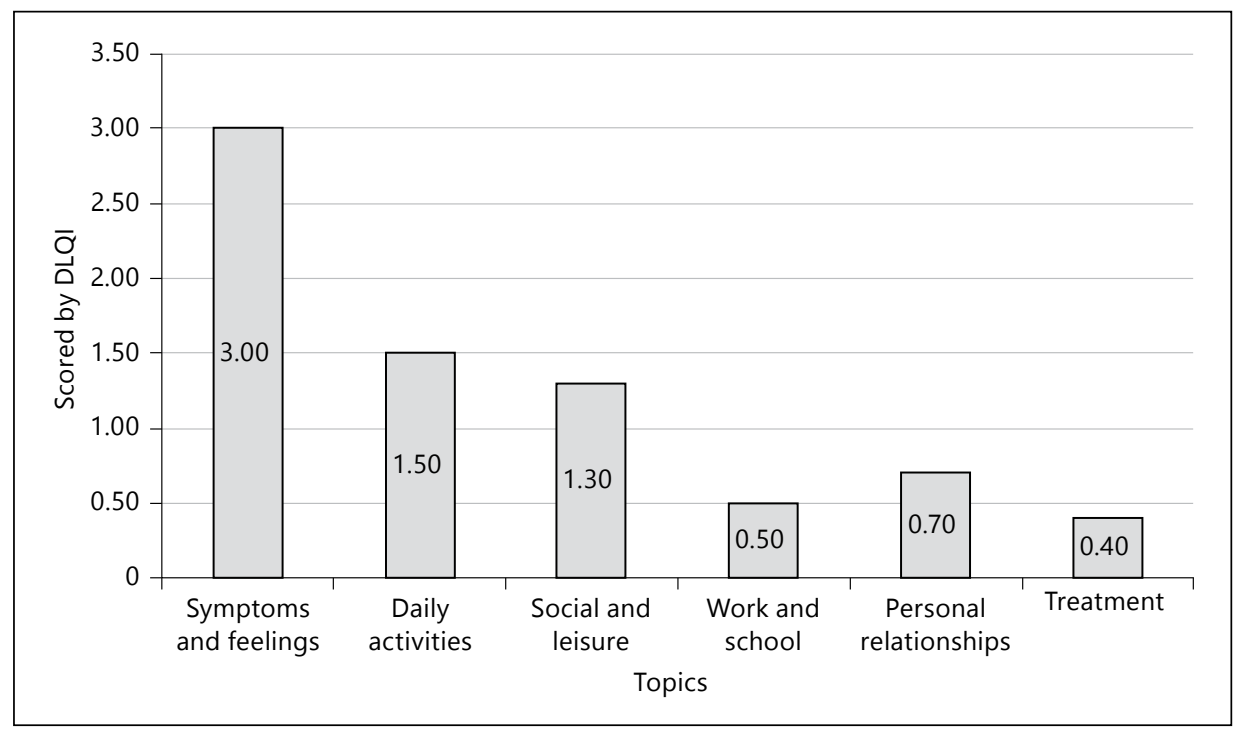

Fig. 2. Tattoos' influence on daily living, measured by the Dermatology Life Quality Index (DLQI).

\section{Conclusion}

Patients with chronic tattoo reactions are heavily burdened by itch, while stinging and pain have a moderate impact on their daily lives. The ISS and DLQI scores are comparable to those of cumber- some and widespread dermatological diseases, e.g. psoriasis, eczema and chronic pruritus. Chronic tattoo reactions should be ranked as a cumbersome dermatological disease and given priority attention and qualified treatment by the public health care system.

\section{References}

1 Hutton Carlsen K, Serup J: Photosensitivity and photodynamic events in black, red and blue tattoos are common: a 'Beach Study'. J Eur Acad Dermatol Venereol 2013, DOI: 10.1111/jdv.12093.

$>2$ Høgsberg T, Hutton Carlsen K, Serup J: High prevalence of minor symptoms in tattoos among a young population tattooed with carbon black and organic pigments. J Eur Acad Dermatol Venereol 2012;27:846-852.

3 Serup J, Hutton Carlsen K: Patch test study of 90 patients with tattoo reactions: negative outcome of allergy patch test to baseline batteries and culprit inks suggests allergen(s) are generated in the skin through haptenization. Contact Dermatitis 2014;71:255-263.
4 Zachariae R, Claus O, Zachariae C, Lei U, Pedersen AF: Affective and sensory dimensions of pruritus severity: associations with psychological symptoms and quality of life in psoriasis patients. Acta Derm Venereol 2008;88:121-127.

5 Majeski CJ, Johnson JA, Davidson SN, Lauzon CJ: Itch Severity Scale: a selfreport instrument for the measurement of pruritus severity. Br J Dermatol 2007; 156:667-673.

6 Finlay AY, Khan GK: Dermatology Life Quality Index (DLQI): a simple practical measure for routine clinical use. Clin Exp Dermatol 1994;19:210-216.
7 Finlay AY, Khan GK: Dermatology Life Quality Index: information \& conditions concerning use. 1992. http://www. dermatology.org.uk/quality/dlqi/ quality-dlqi-info.html (accessed November 8, 2014).

8 Zachariae R, Lei U, Haedersdal M, Zachariae C: Itch severity and quality of life in patients with pruritus: preliminary validity of a Danish adaptation of the Itch Severity Scale. Acta Derm Venereol 2012;92:508-514.

9 Zachariae R, Zachariae C, Ibsen H, Mortensen JT, Wulf HC: Dermatology life quality index: data from Danish inpatients and outpatients. Acta Derm Venereol 2000;80:272-276. 
10 Agner T, Andersen KE, Brandao FM, Bruynzeel DP, Bruze M, Frosch P, Goncalo M, Goossens A, Le Coz CJ, Rustemeyer T, White IR, Diepgen T; EECDRG: Hand eczema severity and quality of life: a cross-sectional, multicentre study of hand eczema patients. Contact Dermatitis 2008;59:43-47.

11 Kim DH, Li K, Seo SJ, Jo SJ, Yim HW, Kim CM, Kim KH, Kim do W, Kim MB, Kim JW, Ro YS, Park YL, Park CW, Lee SC, Cho SH: Quality of life and disease severity are correlated in patients with atopic dermatitis. J Korean Med Sci 2012;27:1327-1332.
2 An JG, Liu YT, Xiao SX, Wang JM, Geng SM, Dong YY: Quality of life of patients with neurodermatitis. Int J Med Sci 2013;10:593-598.

13 Karelson M, Silm H, Kingo K: Quality of life and emotional state in vitiligo in an Estonian sample: comparison with psoriasis and healthy controls. Acta Derm Venereol 2013;93:446-450.
14 Tang MM, Chang CC, Chan LC, Heng A: Quality of life and cost of illness in patients with psoriasis in Malaysia: a multicenter study. Int J Dermatol 2013;52: 314-322.

15 Hutton Carlsen K, Serup J: Patients with tattoo reactions have reduced quality of life and suffer from itch. Dermatology Life Quality Index (DLQI) and Itch Severity Score (ISS) measurements. Skin Res Technol 2015;21:101-107.

Katrina Hutton Carlsen

Department of Dermatology D, Bispebjerg University Hospital

Bispebjerg Bakke 23

DK-2400 Copenhagen NV (Denmark)

E-Mail katrinahuttoncarlsen@hotmail.com 